\section{Stylonichia mytilus and the Lunar Periods}

DURING a recent investigation at the University Botany School, Cambridge, of the association of ciliate protozoa and bacteria in a chalk stream, Styl nichia mytilus in common with other ciliate protozoa exhibited a series of 'pulses', or maximum waves when it spread down the stream, and minimum phases when it could be found only in the upper reaches. Forms in binary fission or conjugation were sometimes noted at the end of the maximum phase, whereas the minimum phase was generally marked by the appearance of small forms. The pulse of Stylonichia could not be related to any constant elimatic or physical factor (rainfall, $p H$, temperature, etc.) or to total bacterial numbers at $22^{\circ} \mathrm{C}$. or any particular group of bacteria. However, the maximum phase often occurred about a week to ten days after one of the lunar periods. From May to December 1947, the maximum phases occurred on twelve out of thirteen occasions a week to ten days after a new moon or a full moon : the greatest maximum peak was attained nine days after a new moon in July and four days after a full moon in December. By contrast with the dry weather and clear nights of 1947, the year 1948 was wet and dull. Stylonichia was only recorded between May and September, yet on five occasions it reached its maximum phase on an average eight days after a new or a full moon.

In the inquiry, samples for protozoological examination were always withdrawn from the stream opposite twelve fixed points, and an arbitrary estimation made of the numbers in not less than six drops of uniform size. A statistical analysis was made of the apparent association between Stylonichia and the lunar periods, and of its association with the full moon, as this was regarded as of particular interest, by the Statistical Laboratory of this University. The arbitrary numbers of Stylonichia were considered in relation to the lunar periodicity in days, and the report of the analysis was as follows. "The observations were classified according to the number of days after the full moon when they were made. Assuming the counts to be distributed in a Poisson distribution a $\chi^{2}$ test was applied to see if the Poisson means were the same at all phases of the moon. In order to make the test valid, it was necessary to group the observations into sets of four days; the mean counts for each group were as follows:

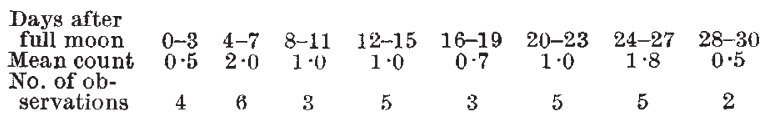

"The value of $\chi^{2}$ was $8 \cdot 42$ on 6 degrees of freedom, not significant. However, when the data were grouped by the number of days to the nearest, not the last, full moon, $\chi^{2}=8 \cdot 15$ was obtained on 2 degrees of freedom, significant at 5 per cent. This suggests that Stylonichia mytilus has a maximum about 4-7 days before and after a full moon, with slight evidence for a drop at the full moon itself. These statements will require more data before they can be made conclusive."

While fully agreeing with the last statement, the statistical analysis was not made until 1949 , a year after the inquiry was completed, and it is perhaps re- markable that so suggestive a decision was reached on field-observations made with quite a different object in view. Alone, the observation might not be of so much interest were it not that Stylonichia mytilus is a diatom-eating ciliate and there is an abundant phytoplankton of diatoms in the stream concerned, which is swift, cold and shallow. Kofoid', in a statistical analysis of the phytoplankton of the Illinois River, records an association between the pulse of Bacillariæ (that is, diatoms) and the lunar periods. He states that the average lag of the phytoplankton pulse after the full moon was $13 \cdot 7$ and 14.8 days respectively and that the correlation of full moon and maximum phase of diatoms was most intimate in the stable conditions of summer when the average of the average monthly lags for all the May-August pulses was 11.9 days. In the absence of other information, it was assumed that in the particular stream examined the Stylonichia were follow. ing a lunar periodicity of diatoms (on which they fed) similar to that described by Kofoid in the Illinois. Maximum phases of the ciliate might follow therefore maximum phases of the diatoms, succeeded by periods of reproductive activity.

I wish to express my thanks to Mr. D. V. Lindley, who made the above statistical analysis.

National Agricultural Advisory Service, ERnest Gray Anstey Hall

Trumpington, Cambridge. Sept. 13.

${ }^{ }$Kofoid, C. A., Bull. Ill. Lat. Nat. Hist., 6, 95 (1903).

\section{Duration of the Larval Period in the Brook Lamprey (Lampetra planeri)}

Although the question of growth and the duration of the larval period in the brook lampreys has received considerable attention, the conclusions reached have been indecisive. In these animals, which spend the whole of their life in fresh-water, the problem is simplified in so far as the growth period ends at metamorphosis with the closure of the fore-gut and the cessation of feeding. Sexual maturity is reached in the first spring following metamorphosis, when the adult spawns and dies.

The most complete of recent work on this subject is that of Knowles in Italy, Schultze ${ }^{2}$ in Michigan and Ivanova-Berg ${ }^{3}$ in the Gulf of Finland. Estimates of the total life-span as given by these authors are three, three to four and five years respectively. For L. planeri in the British Isles, the only similar study, so far as is known, is my own 4 , based on collections of larve and adults from streams in Somerset. The con-

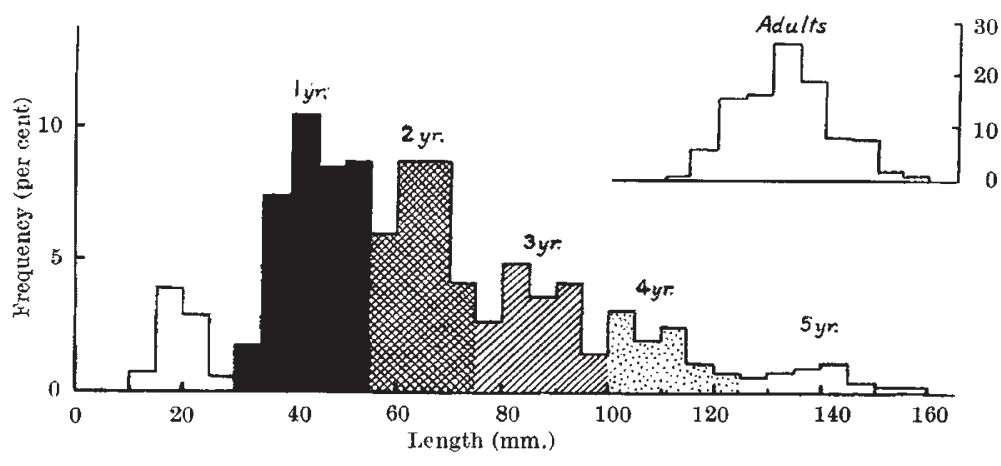

